Reprod. Nutr. Dévelop., 1982, 22 (6), 951-958.

\title{
Relations entre l'anneau postnucléaire, la chromatine et l'enveloppe nucléaire des spermatides de Bélier. Conception d'un modèle partiel pour expliquer les déplacements de la manchette
}

\section{J.-L. COURTENS}

avec la collaboration technique de Jitka DURAND et Bernadette DELALEU

Station de Physiologie de la Reproduction, I.N.R.A. Nouzilly, 37380 Monnaie, France

Summary. Relationships between the postnuclear band, chromatin and nuclear envelope of ram spermatids. A proposal of a partial model to explain the pulling of the manchette.

After alkaline extraction, the postnuclear band appeared to be composed of two parts, the intranuclear part contiguous to the chromatin and the extranuclear part apposed to the external membrane of the nuclear envelope. This membrane was discontinuous inside the postnuclear band, revealing that the band might be a zone of nuclear envelope remodeling. A model is proposed to explain the pulling of the manchette by intranuclear forces and the formation of the redundant nuclear envelope during spermiogenesis.

\section{Introduction.}

L'anneau postnucléaire entoure le noyau des spermatides, immédiatement derrière l'acrosome. II réunit l'enveloppe nucléaire à la membrane plasmique (Courtens et al., 1976) et joue un rôle fondamental dans les processus de différenciation des spermatides, puisqu'il entraîne la manchette avec lui quand il se déplace vers l'arrière (Courtens, 1978). Des travaux précédents (Courtens et Loir, 1975 a et b ; 1981 a) ont montré qu'il glissait à la surface du noyau, à la limite entre deux zones nucléaires cytochimiquement distinctes: celle qui contient encore des histones et des nucléoprotéines spécifiques des spermatides, et celle qui n'en contient plus. Tout se passe donc comme si des modifications intranucléaires pouvaient " induire " le mouvement d'un organite extranucléaire à travers l'enveloppe nucléaire. L'anneau postnucléaire n'est généralement pas visible quand les techniques d'étude ultrastructurale classiques sont utilisées. C'est pourquoi il n'a été signalé que rarement (Sandoz, 1970 ; Lalli et Clermont, 1981). Il n'est décrit qu'à partir de techniques cytochimiques qui le mettent mieux en 
évidence (Courtens et Loir, 1975 a, b ; Courtens et al., 1976). Toutefois, l'encombrement et la petite taille de la zone cytoplasmique où il se trouve, ainsi que la trop faible résolution des techniques cytochimiques, ne permettent pas d'étudier cet anneau de manière très précise. Pour mieux en cerner l'organisation, nous avons eu recours à une technique d'extraction des constituants cellulaires par un tampon à $\mathrm{pH}$ élevé, qui modifie l'ionisation des protéines et favorise le « relargage » des plus électropositives. Le contraste général des ultrastructures est notablement abaissé par ce procédé. Celui de l'anneau postnucléaire, ou celui des portions d'anneau qui persistent, devient ainsi égal à celui des autres organites voisins. Il est alors possible de l'observer sans avoir recours à la cytochimie. Seule la période de déplacement de l'anneau est étudiée ici chez le Bélier (les spermatides au stade 12 sont donc les seules considérées).

\section{Matériel et méthodes.}

Des pièces de testicules de Bélier de $3 \mathrm{~mm}$ de côté sont fixées pendant $4 \mathrm{~h}$ par 4 p. 100 de glutaraldéhyde dissous dans du tampon borate $0,1 \mathrm{M}, \mathrm{pH}$, ou du tampon $\mathrm{NaOH} / \mathrm{PO}_{4} \mathrm{Na}_{2} \mathrm{H}, 0,05 \mathrm{M}, \mathrm{pH} 11$ ou 13. Elles sont ensuite lavées à l'eau distillée, puis sont post fixées par 2 p. 100 de tétroxyde d'osmium dissous dans le tampon phosphate ou cacodylate, $0,1 \mathrm{M}, \mathrm{pH} 7,3$, avant d'être incluses en Epon 812. Les coupes ultrafines sont contrastées à l'acétate d'uranyle aqueux ou méthanolique, puis au citrate de plomb.

\section{Résultats}

L'extraction des pièces, jugée sur l'aspect et la colorabilité des noyaux de spermatides au stade 12 est d'autant plus importante que le $\mathrm{pH}$ du fixateur est plus élevé (fig. 1 et 2), mais à $\mathrm{pH} \mathrm{9,} \mathrm{la} \mathrm{chromatine} \mathrm{révèle} \mathrm{une} \mathrm{ultrastructure} \mathrm{simi-}$ laire à celle observée aux $\mathrm{pH}$ neutres : après coloration par l'acétate d'uranyle aqueux et par le citrate de plomb, elle est uniformément dense en fin de stade 12. A pH 11 et 13, par contre, la colorabilité nucléaire est moindre (fig. 1) ; l'aspect de la chromatine est comparable à celui observé aux stades précédents (10 et 11) après fixation à $\mathrm{pH}$ neutre. De grosses fibres de chromatine, alignées dans le sens antéro-postérieur, et des images de granules, pouvant résulter de la coupe transversale des fibres, sont réunies par de multiples fibres plus fines (fig. 1).

L'enveloppe nucléaire présente deux aspects différents, selon qu'elle est antérieure ou non à l'anneau postnucléaire. Dans la région antérieure, ses 2 membranes sont très proches, et souvent - mais pas toujours - indistinctes après l'extraction (fig. 1) à pH élevé. Par contre, dans la région postérieure, les 2 membranes sont séparées par un espace plus grand. Après l'extraction, l'enveloppe nucléaire est parfois discontinue, mais elle demeure réunie à la chromatine par des fibres très fines (fig. 1). 
L'anneau postnucléaire est formé de 2 sous-unités, séparées par l'espace périnucléaire. De l'intérieur vers l'extérieur, on observe successivement :

a) une substance d'aspect amorphe, en contact étroit avec la chromatine ;

b) une couche très mince de substance plus opaque aux électrons, formée par les 2 membranes de l'enveloppe nucléaire " antérieure », ou (et) la membrane interne de l'enveloppe nucléaire « postérieure » ;

c) une couche claire, d'épaisseur constante, correspondant à l'espace périnucléaire de l'enveloppe " postérieure ». Cette couche nucléaire est en effet bordée du côté externe par la membrane externe de l'enveloppe nucléaire " postérieure";

d) une couche de matériel amorphe, extranucléaire, est reliée à la lame postacrosomique en formation (fig. 1 et 2), à la membrane plasmique, et à l'anneau nucléaire, par des agrégats mal définis après l'extraction. Cette dernière couche correspond à la seule portion d'anneau décrite à ce jour (Courtens et Loir, $1975 \mathrm{~b}$; Courtens et al., 1976).

\section{Discussion.}

C'est au stade 12, chez le Bélier, que débute la condensation de la chromatine et que disparaissent définitivement les histones et les nucléoprotéines intermédiaires, spécifiques des spermatides. En leurs lieux et places, apparaît la protamine. Dans ce travail, nous $n^{\prime}$ avons pas cherché quels éléments étaient perdus par l'extraction, toutefois, le "rajeunissement " apparent des noyaux après l'extraction révèle sans doute que le matériel extrait des noyaux au stade 12 masque habituellement une structure déjà présente dans les noyaux applatis mais pas encore condensés. Des résultats identiques, obtenus en traitant les spermatides par des solutions salines de force ionique importante (Loir, communication personnelle), en absence de protéolyse, révèlent que les substances perdues n'étaient fixées à la chromatine que par des liaisons électrostatiques. Le démasquage de cette structure nucléaire au stade 12 présente un autre intérêt, car il révèle que l'anneau postnucléaire est un organite à la fois intra et extra-nucléaire ; et qu'à son niveau l'enveloppe nucléaire présente une structure inhabituelle en ce sens que la membrane externe $y$ est interrompue. Cette discontinuité (schéma) éclaire des observations précédentes d'un jour nouveau : la structure de l'enveloppe est différente en amont et en aval de I'anneau. En amont, elle est dépourvue de pores nucléaires (Chemes et al., 1978) ; elle est appliquée étroitement à la chromatine ; elle est formée de 2 membranes très proches (Sandoz, 1970) ; elle est traversée par de très nombreuses fibres qui réunissent la chromatine à la substance périnucléaire (Courtens et Loir, 1981 b) ; et elle est colorable par l'acide phosphotungstique alcoolique (Courtens et Loir, 1975 a).

En aval, au contraire, elle contient tous les pores nucléaires (Wischnitzer, 1970 ; Gordon, 1972) ; elle est séparée de la chromatine (Loir et Courtens, 1979) ; elle est formée de 2 membranes séparées par un espace périnucléaire important (Sandoz, 1970 et 1974) ; elle est traversée par quelques fibres qui réunissent la chromatine à la manchette (Courtens et Loir, 1981 b) ; elle n'est pas 


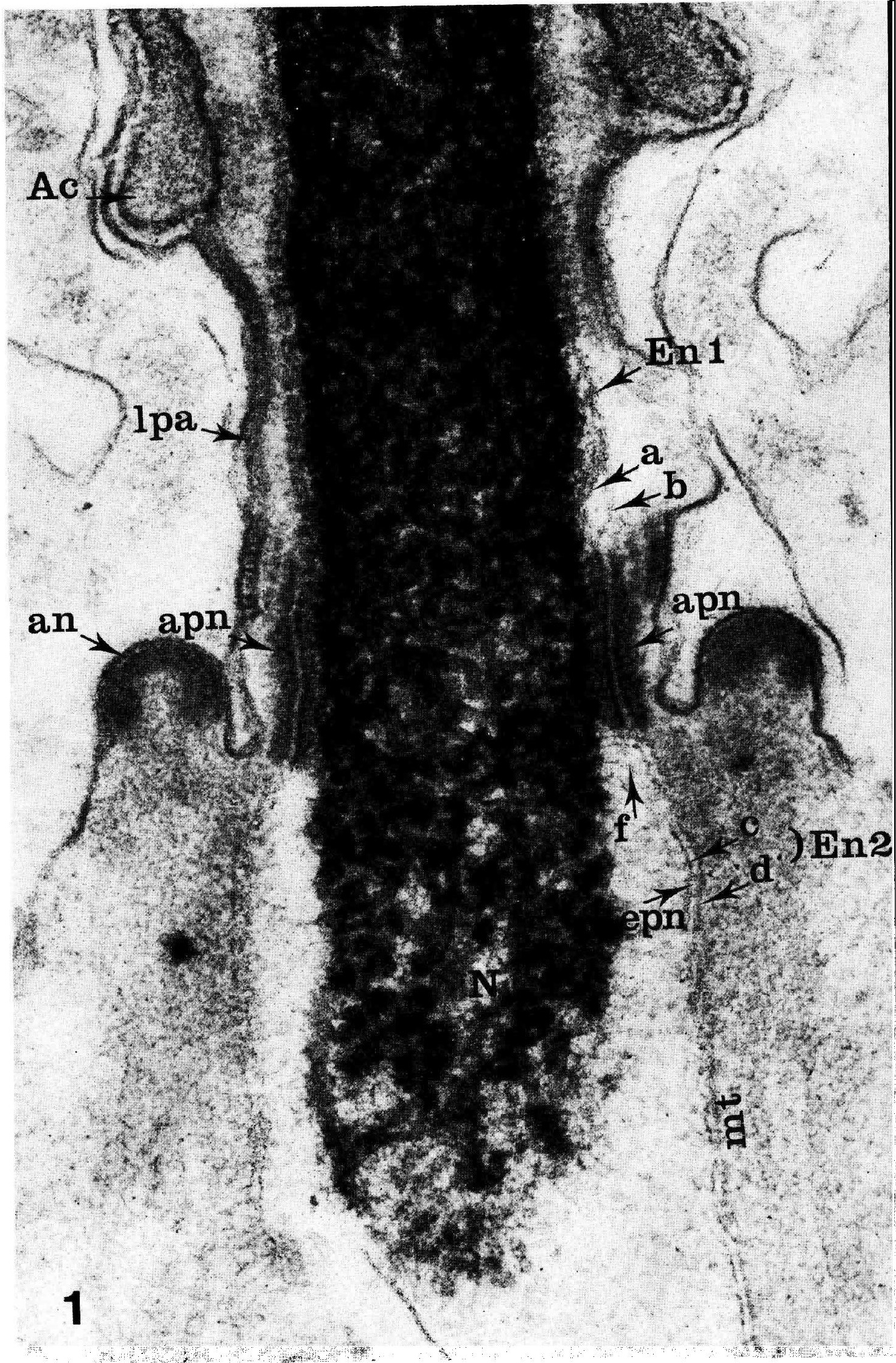




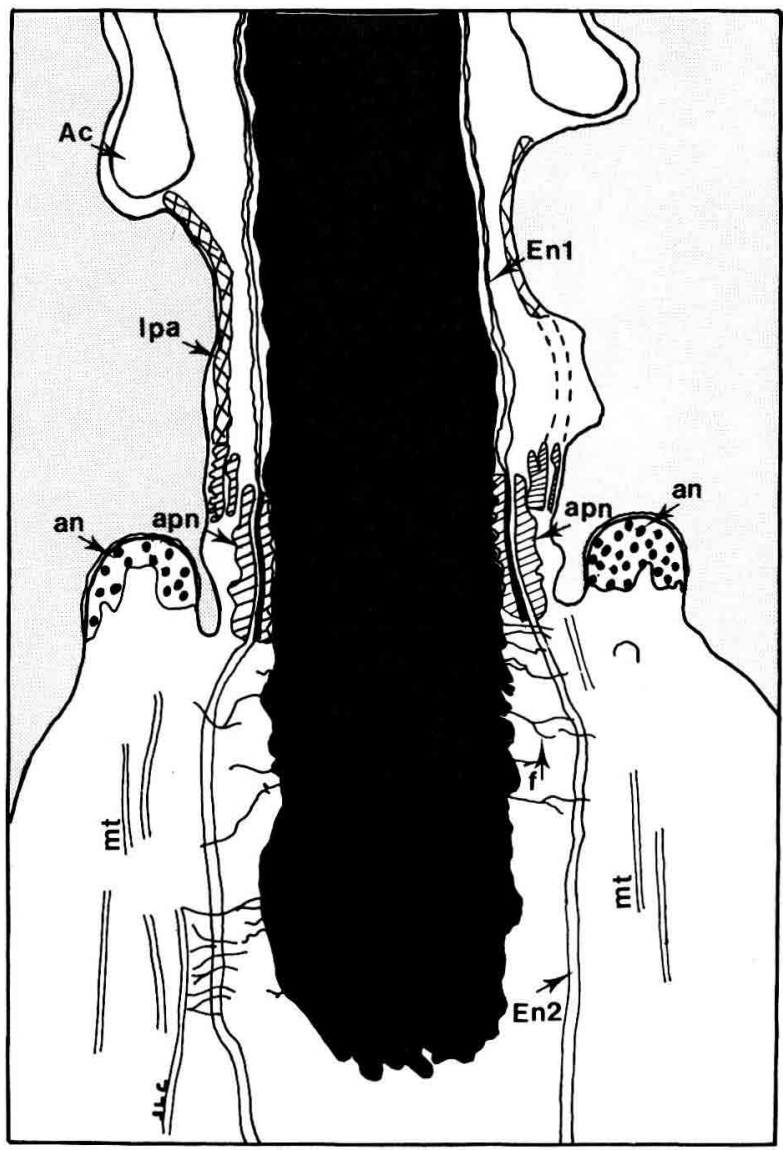

FIG. 1 et schéma. - Spermatide de Bélier au milieu du stade 12.

L'anneau postnucléaire (apn) a quitté sa position postacrosomique ( $\mathrm{Ac}=$ acrosome) pour glisser vers l'arrière du noyau ( $N$ ). L'enveloppe nucléaire antérieure (EN I) est formée de 2 membranes a et $b$ très proches. Elles sont parfois séparées après l'extraction à pH basique. L'enveloppe nucléaire postérieure (EN 2) est constituée par des membranes $c$ et $d$ séparées par un " espace périnucléaire " (epn). L'anneau postnucléaire est situé de part et d'autre des membranes c et $d$. II est en contact avec la chromatine et est également relié à la lame postacrosomique (LPA) qui borde la membrane plasmique en arrière de l'acrosome $(A c)$. Les microtubules de la manchette (mt) sont implantés dans l'anneau nucléaire (an). Des fibres issues de la chromatine (f) rejoignent la manchette à travers l'enveloppe nucléaire postérieure. Celle-ci est partiellement extraite par le traitement à pH 13. La chromatine est également moins condensée que chez les témoins au même stade. Son aspect est représentatif de spermatides au stade 11. $\times 62000$. 


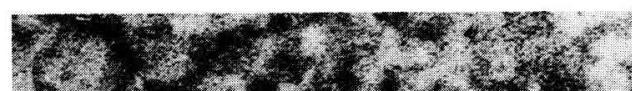
$6+204+1 x^{2}$ sortats

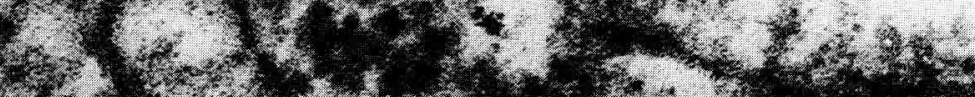
2.

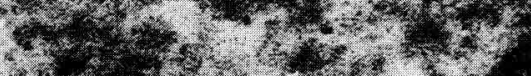
(6)

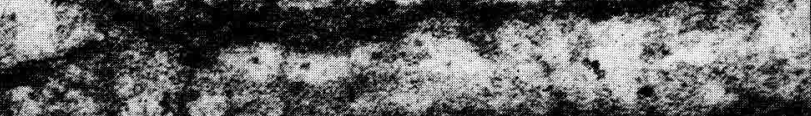
c

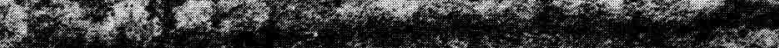

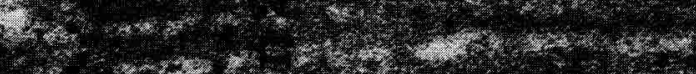

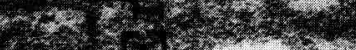

10.7 m.

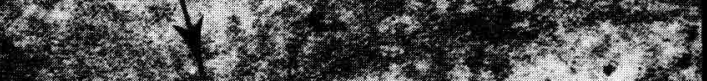

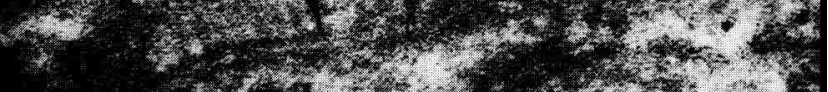

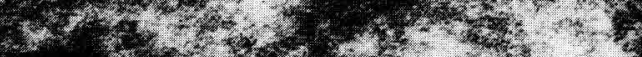
- 640

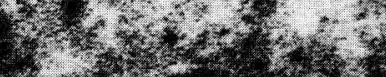
Sor $2 .-2,5$

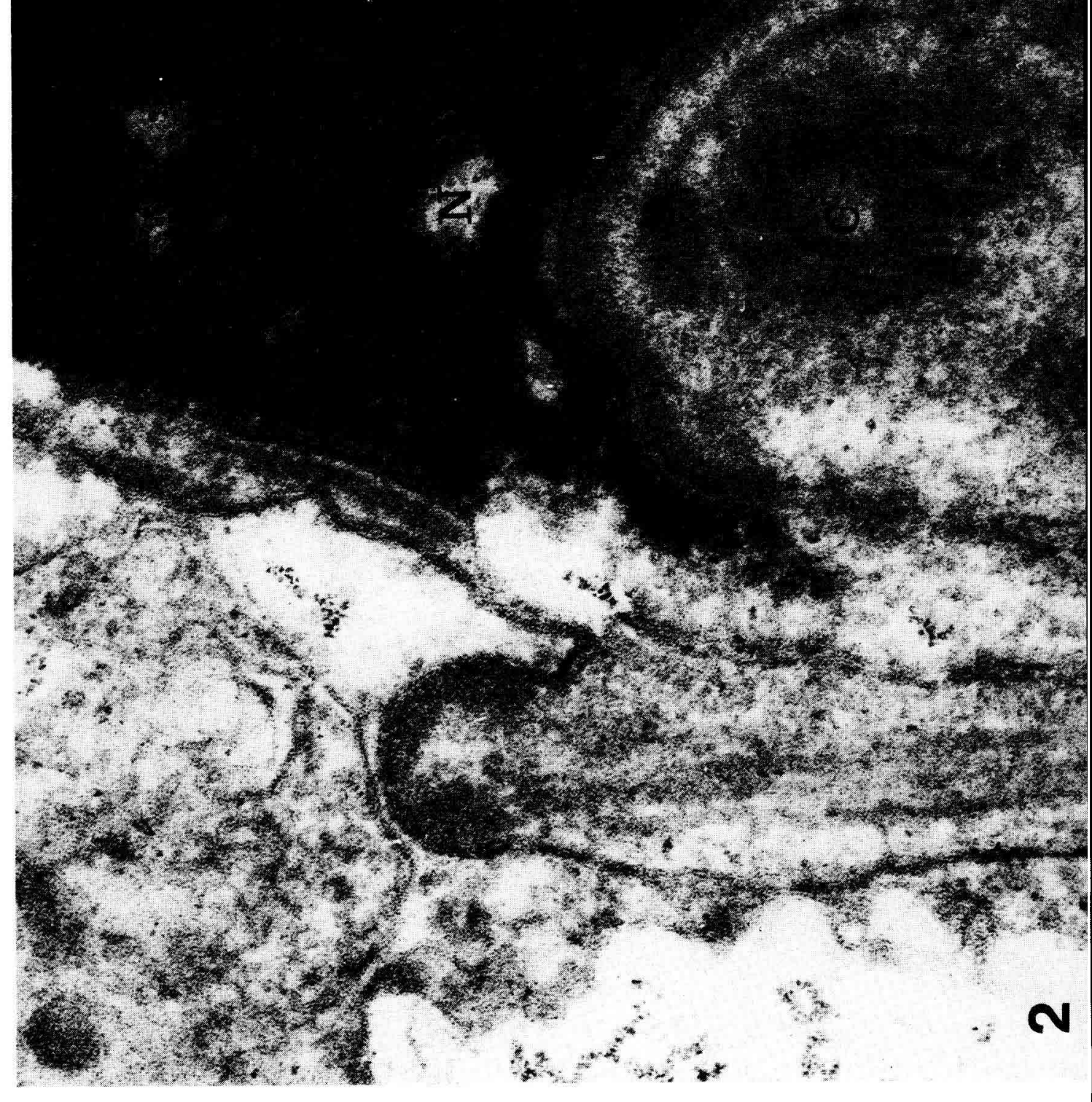


colorable par l'acide phosphotungstique alcoolique (Courtens et Loir, 1975 a). Enfin, elle est fixée à la manchette par de nombreux liens (McKinnon et Abraham, 1972).

Tout ceci semble indiquer que seule la portion d'enveloppe nucléaire postérieure à l'anneau postnucléaire possède les caractères de l'enveloppe nucléaire des jeunes spermatides, alors que celle située en amont présente une structure et une composition différente ou nouvelle. L'anneau postnucléaire est-il le siège de la synthèse d'une nouvelle enveloppe nucléaire ? Ou celui d'un recyclage des éléments de «l'ancienne » enveloppe? L'anneau postnucléaire repousse-t-il devant lui l'enveloppe nucléaire de la jeune spermatide pendant sa migration vers l'arrière?

Ces hypothèses permettraient d'expliquer la formation des membranes nucléaires redondantes quand la manchette glisse vers l'arrière (De Kretzer, 1969 ; Rattner et Brinkley, 1971). Elles permettraient de mieux comprendre comment les fibres, attachées à la fois à la manchette et à la chromatine, glissent vers l'arrière, avec la manchette (Courtens et Loir, 1981 b) à travers "I'ancienne " enveloppe ; comment s'effectue la redistribution des pores nucléaires vers l'arrière pendant la spermiogenèse (Fawcett et Chemes, 1979) et un corrolaire possible : le déplacement des fibres de chromatine attachées aux pores (Nicolini, 1980). Elles permettraient enfin de montrer que, si la manchette est réellement équivalente à un demi-fuseau achromatique (Courtens, 1982 b), sont mouvement vers l'arrière est très différent de celui d'un véritable fuseau (Inoue, 1981) puisqu'il est commandé par le glissement de l'anneau nucléaire, luimême solidaire de l'anneau postnucléaire (Courtens, 1978).

Les observations présentes révèlent que le mouvement de l'anneau postnucléaire - structure à la fois intra et extra-nucléaire, et frontière entre deux zones de l'enveloppe nucléaire - pourrait être commandé par un mécanisme intranucléaire. Il est en effet remarquable de constater que la composition de la chromatine située en amont de l'anneau évolue lorsque l'anneau glisse vers l'arrière chez au moins six espèces (Courtens et Loir, 1981 a). A la nouvelle composition correspond le rapprochement des fibres de chromatine, qui se propage également vers l'arrière (Loir et Courtens, 1979). II semble possible que les modifications physiques de la chromatine puissent induire le mouvement de l'anneau postnucléaire vers l'arrière. La différenciation du noyau pourrait ainsi, en dirigeant celle du cytoplasme adjacent, réaliser une boucle rétroactive, puisque les éléments cytoplasmiques, associés à la manchette, ont une influence primordiale sur la différenciation du noyau (Courtens, 1982 a et b).

Reçu en mars 1982

Accepté en juin 1982

FIG. 2. - Spermatide de Bélier en fin de stade 12.

Extraction à $\mathrm{pH}$ 11. L'anneau postnucléaire (apn) est maintenant arrivé en position distale. Remarquer la discontinuité entre l'enveloppe nucléaire postérieure (I'enveloppe redondante) (EN 2) et l'enveloppe antérieure (EN 1), plus proche de la chromatine. L'extraction du noyau (N) est moins importante qu'à $\mathrm{pH} 13$ (comparer avec la fig. 1 ). $\mathrm{C}=$ centriole ; $\mathrm{mp}=$ membrane plasmique. $\times 84600$. 


\section{Références}

CHEMES H. B., FAWCETT D. W., DYM M., 1978. Unusual features of the nuclear envelope in the human spermatogenic cells. Anat. Rec., 192, 493-512.

COURTENS J. L., 1978. Morphogenesis of postacrosomal cytoplasm in the spermatid head. Experimental study of hypophysectomized and testosterone-injected rams. Ann. Biol. anim. Bioch. Biophys., 18, 1455-1461.

COURTENS J. L., 1982 a. Some nucleocytoplasmic exchanges during spermiogenesis of boar, ram and stallion. Gamete Res., 5, 137-152.

COURTENS J. L., 1982 b. Rôles indirects des microtubules dans la morphogenèse nucléaire des spermatides. Reprod. Nutr. Dévelop., 22 (sous presse).

COURTENS J. L., COUROT M., FLECHON J. E., 1976. The perinuclear substance of boar, bull, ram and rabbit spermatozoa. J. Ultrastruct. Res., 57, 54-64.

COURTENS J. L., LOIR M., 1975 a. Mise en évidence par cytochimie ultrastructurale de la migration des histones riches en lysine au cours de la spermiogenèse du bélier. J. Microscopie Biol. cell., 24, 249-258.

COURTENS J. L., LOIR M., 1975 b. Mise en évidence par cytochimie ultrastructurale de l'évolution des organites cytoplasmiques contemporaine de la perte des histones somatiques au cours de la spermiogenèse du bélier. J. Microscopie Biol. cell., 24, 259-270.

COURTENS J. L., LOIR M., 1981 a. A cytochemical study of nuclear changes in boar, bull, goat, rat and stallion spermatids. J. Ultrastruct. Res., 74, 327-340.

COURTENS J. L., LOIR M., 1981 b. The spermatid manchette of mammals : formation and relations with the nuclear envelope and the chromatin. Reprod. Nutr. Dévelop., 21, 467-477.

DE KRETZER D. M., 1969. Ultrastructural features of human spermiogenesis. Z. Zellforsch., 98, 477-505.

FAWCETT D. W., CHEMES H. E., 1979. Changes in distribution of nuclear pores during differentiation of the male germ cells. Tissue Cell., 11, 147-162.

GORDON M., 1972. The nuclear envelope in guinea pig spermiogenesis. Anat. Rec., 173, 45-56.

INOUE S., 1981. Cell division and the mitotic spindle. J. Cell. Biol., 91, 1315-1475.

LALLI M., CLERMONT Y., 1981. Structural changes of the head components of the rat spermatid during late spermiogenesis. Amer. J. Anat., 160, 419-434.

LOIR M., COURTENS J. L., 1979. Nuclear reorganization in ram spermatids. J. Ultrastruct. Res., 67, 309-324.

McKINNON E. A., ABRAHAM P. J., 1972. The manchette in stage 14 rat spermatids: a possible structural relationship with the redundant nuclear envelope. Z. Zelfforsch., 124, $1-11$.

NICOLINI C., 1980. Nuclear morphometry, quinternary chromatin structure and cell growth. J. Submicrosc. Cytol., 12, 475-505.

RATTNER J.. B., BRINKLEY B. R., 1971. Ultrastrúcture of mammalian spermiogenesis. II. Elimination of the nuclear membrane. J. Ultrastruct. Res., 36, 1-7.

SANDOZ D., 1970. Evolution des ultrastructures au cours de la formation de l'acrosome du spermatozoïde chez la souris. J. Microscopie, 9, 535-558.

SANDOZ D., 1974. Modifications in the nuclear envelope during spermiogenesis of Discoglossus pictus (anuran Amphibia). J. Submicr. Cytol., 6, 399-419.

WISCHNITZER S., 1970. The annulate lamellae. Int. Rev. Cytol., 27, 65-100. 